\title{
Intramucosal carcinoma of the gastric antrum complicating Menetrier's disease
}

\author{
GORDON M WOOD, CHRISTINE BATES, RICHARD C BROWN, MONTY S LOSOWSKY
}

From the Departments of Medicine and Pathology, St James's University Hospital, Beckett Street, Leeds LS9 $7 T F$

SUMMARY A 48-year-old man presented with haematemesis and was found to have Menetrier's disease. Three and a half years later early gastric cancer was detected at routine follow-up endoscopy. This is the first report of the development of early gastric cancer during follow-up in a case of Menetrier's disease. It is probable that patients with Menetrier's disease are at increased risk of gastric cancer, and should have regular endoscopic follow-up with gastric biopsy.

The controversy over the possibility that Menetrier's disease predisposes to gastric cancer is assuming greater importance now that more cases of Menetrier's disease are managed without gastric resection.

We report a patient who developed an early gastric cancer three and a half years after Menetrier's disease was first diagnosed, and review present knowledge of the relationship between the two conditions.

\section{Case report}

The patient first presented with haematemesis at the age of $48 \mathrm{yr}$. He had had mild non-specific dyspepsia for many years. There were no abnormal findings on examination, apart from melaena. Barium meal demonstrated large mucosal folds along the greater curvature of the stomach and poor mucosal coating with barium, due to copious mucus secretion. The radiological diagnosis was a greater curve gastric carcinoma. Gastroscopy showed that the enlarged folds on the greater curve were composed of thickened, oedematous gastric mucosa with superficial erosions. The endoscopic diagnosis was Menetrier's disease with a superimposed erosive gastritis.

Histological sections of gastric mucosal biopsies showed an unusually abundant gastric mucosa, with disruption and loss of continuity of the muscularis mucosae. The glands were lined by mucous cells and some showed cystic dilatation; parietal cells were also present indicating body mucosa. The superficial mucosa was infiltrated by lymphocytes and neu- trophils (Fig. 1). The histological diagnosis was Menetrier's disease with a chronic superficial gastritis. There was no evidence of neoplastic change.

The patient had no further gastrointestinal bleeding during this first admission. He continued to have mild dyspepsia with little relief from antacids. Twelve months later he suffered an extensive inferior myocardial infarction. After a further 13 months, he was readmitted with epigastric pain and melaena. Gastroscopy again showed large folds of mucosa in the fundus and body of the stomach. As before, there were scattered mucosal erosions, some of which were bleeding. Biospy of the gastric antrum showed a chronic superficial gastritis with no evidence of neoplasia. Cimetidine was commenced at a dose of $1 \mathrm{~g} /$ day with rapid symptomatic relief and the patient was followed by regular endoscopy.

After four months treatment with cimetidine, the erosions had healed but large gastric folds were still present. There was no further change at follow-up endoscopy 11 months later. On this occasion the body and antrum were again biopsied. The body mucosa showed similar features to the first biopsy described above. There was no evidence of malignant change. Two of the five antral biopsies, however, showed large hyperchromatic, pleomorphic epithelial cells with occasional mitotic figures, the appearances being those of gastric adenocarcinoma. The patient was readmitted to hospital and cimetidine was discontinued. Examination revealed no abnormal findings and investigations included a serum albumin of $43 \mathrm{~g} / \mathrm{l}$, a basal acid output of $0 \cdot 19$ $\mathrm{mmol} / \mathrm{h}$ and peak acid output of $18.8 \mathrm{mmol} / \mathrm{h}$ after pentagastrin. Double contrast barium meal confirmed coarse gastric mucosal folds along the 
greater curve with a thickened stomach wall but no abnormality was found in the antrum (Fig. 2).

At laparotomy the abdominal viscera appeared normal, apart from the stomach which had a normal serosa but a grossly thickened and lumpy wall. A two-thirds Polya gastrectomy was performed. The patient had a satisfactory convalescence apart from an episode of multifocal ventricular ectopic beats in the immediate post-operative period. He remains well 18 months later. Follow-up gastroscopy and biopsy of the gastric remnant has shown no evidence of recurrent carcinoma.

The gastrectomy specimen was opened along the greater curvature which measured $24 \mathrm{~cm}$ in length. Large folds of mucosa up to $1 \mathrm{~cm}$ wide were present in the body and fundus and were most prominent along the greater curve. The antrum was macroscopically normal apart from slight nodularity of the mucosa (Fig. 3). Histologically, the body of the

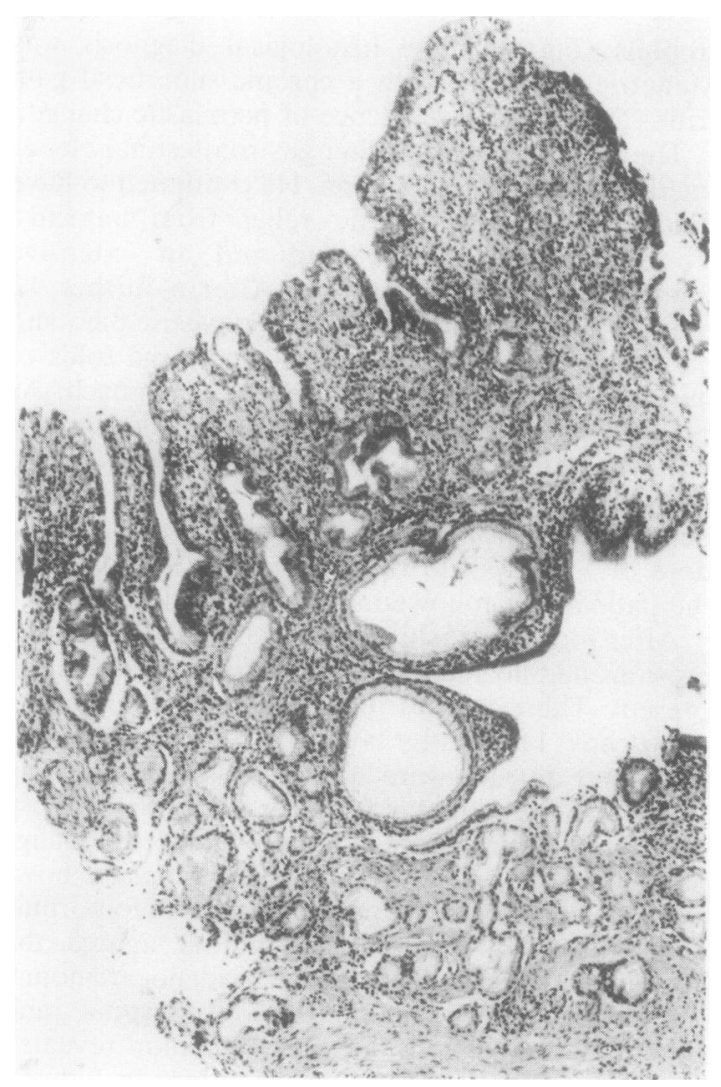

Fig. 1 Original gastroscopic biopsy showing gastric mucosa with disruption of muscularis mucosae, cystic glands lined by mucous cells and a superficial infiltrate of lymphocytes and neutrophils. Haematoxylin and eosin $\times$ 80. stomach showed features of advanced Menetrier's disease. The gastric mucosa was heaped up, forming giant rugae (Fig. 4). The glands were long and straight with the usual complement of gastric and parietal cells, but in many areas cystic dilatation of the basal portion of the glands had occurred. In places, the glands herniated through the muscularis mucosae which was frayed and disrupted. Metaplasia of the epithelium to mucus-secreting goblet cells had occurred in the cystic portions of the glands. Plasma cells and polymorphonuclear leucocytes were present throughout the lamina propria but they were especially prominent in the superficial areas. Nine blocks were taken from the body of the stomach with no evidence of malignancy. One block out of 15 taken from the gastric antrum showed intramucosal carcinoma, spreading superficially. There was no invasion beyond the lamina propria (Fig. 5). Fourteen lymph nodes were examined and found to be free of metastatic deposits. The antral mucosa in the area of carcinomatous change also showed features of Menetrier's disease with cystic glands, superficial inflammation and disrupted muscularis mucosae but the mucosa itself was relatively thin. Other sections from the antrum showed similar features of the disease which were less marked, though inflammation of the lamina propria was noted in all areas. A few small areas of intestinal metaplasia were observed.

\section{Discussion}

The patient described above had the clinical, radiological and endoscopic features of Menetrier's disease when he first presented. ${ }^{12}$ The serum albumin is usually low in this condition but is more than $35 \mathrm{~g} / 1$ in $20 \%$ of cases. ${ }^{1}$ Gastric biopsies at that time showed typical histological changes of the disease, with hypertrophy of the gastric glands and cystic dilatation of their basal portions, replacement of parietal cells by mucus cells and a moderate inflammatory infiltrate in the lamina propria. ${ }^{1-3}$ The biopsies were adequate to make a confident diagnosis of Menetrier's disease as they included the muscularis mucosae. Full-thickness biopsies from the gastrectomy specimen later confirmed this conclusion and also demonstrated microscopc features of Menetrier's disease in the antrum; gross antral involvement in this condition is rare but has been described. ${ }^{45}$ No other cause for giant gastric folds was found. There is thus no doubt that this patient had Menetrier's disease when he first presented with a haematemesis.

Carcinoma was found in antral biopsies taken three and a half years after the original diagnosis of Menetrier's disease, and early gastric cancer was 


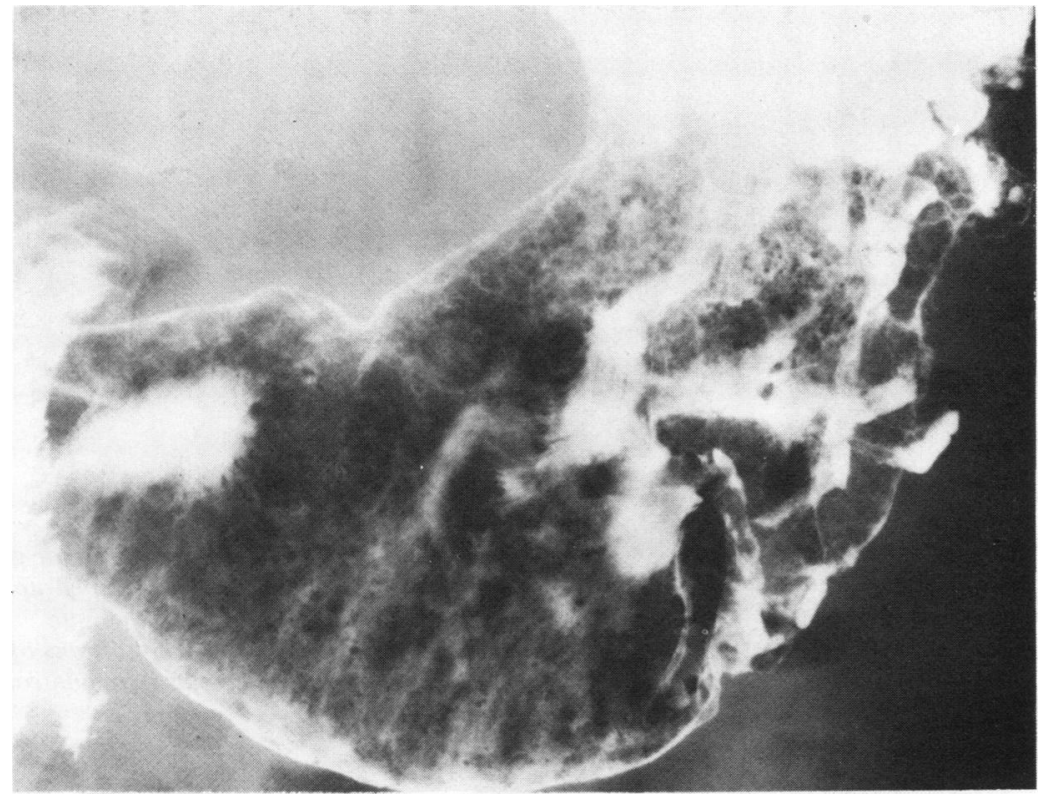

Fig. 2 The double contrast barium meal shows giant folds on the greater curvature of the body of the stomach but the antrum appears normal. confirmed in the gastrectomy specimen. Although early gastric cancer may remain confined to the mucosa for 3-4 $\mathrm{yr}^{6}{ }^{6}$ we had taken antral biopsies on two occasions during follow-up in our patient, with no evidence of malignancy. We therefore believe it to be very likely that early gastric cancer developed after Menetrier's disease in this case.
When Menetrier first described "polyadenomes en nappe", he considered the lesion to be benign, but wrote "... it does have affinity with a more malignant state and these lesions may be transformed into epitheliomas and cancers".? Although there have been more than 30 subsequent case reports of gastric carcinoma found in association

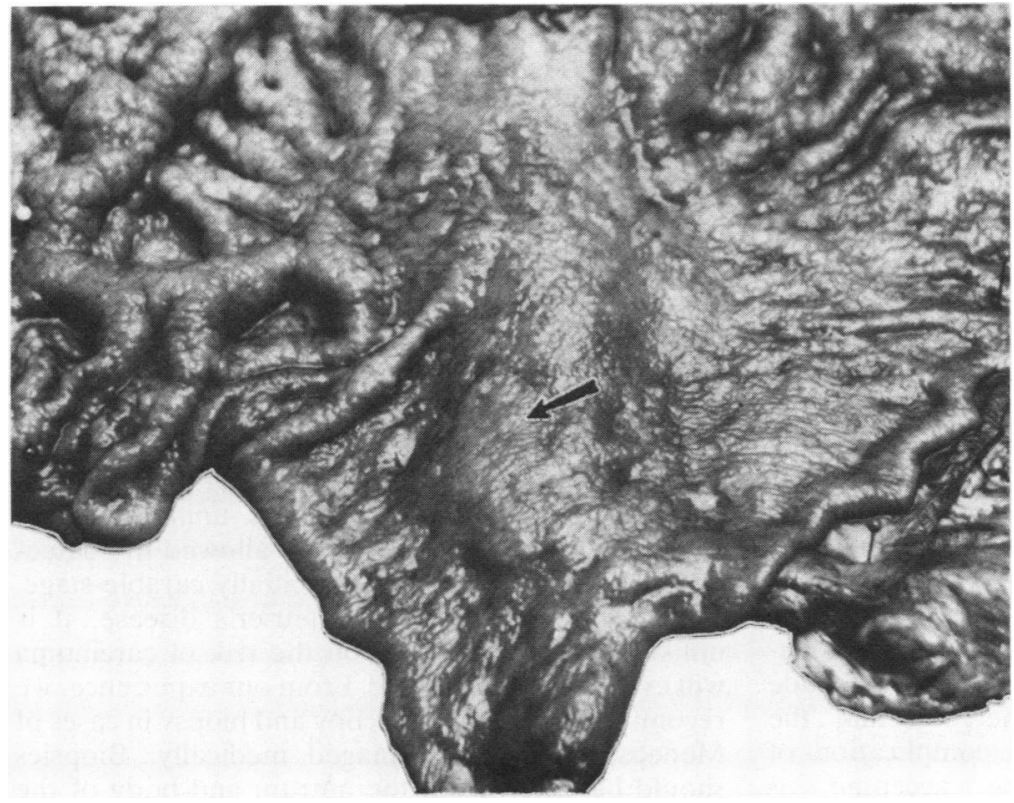

Fig. 3 The gastrectomy specimen opened along the greater curve showing giant folds in the body of the stomach with sparing of the antrum. The arrow indicates the site of the intramucosal carcinoma. 


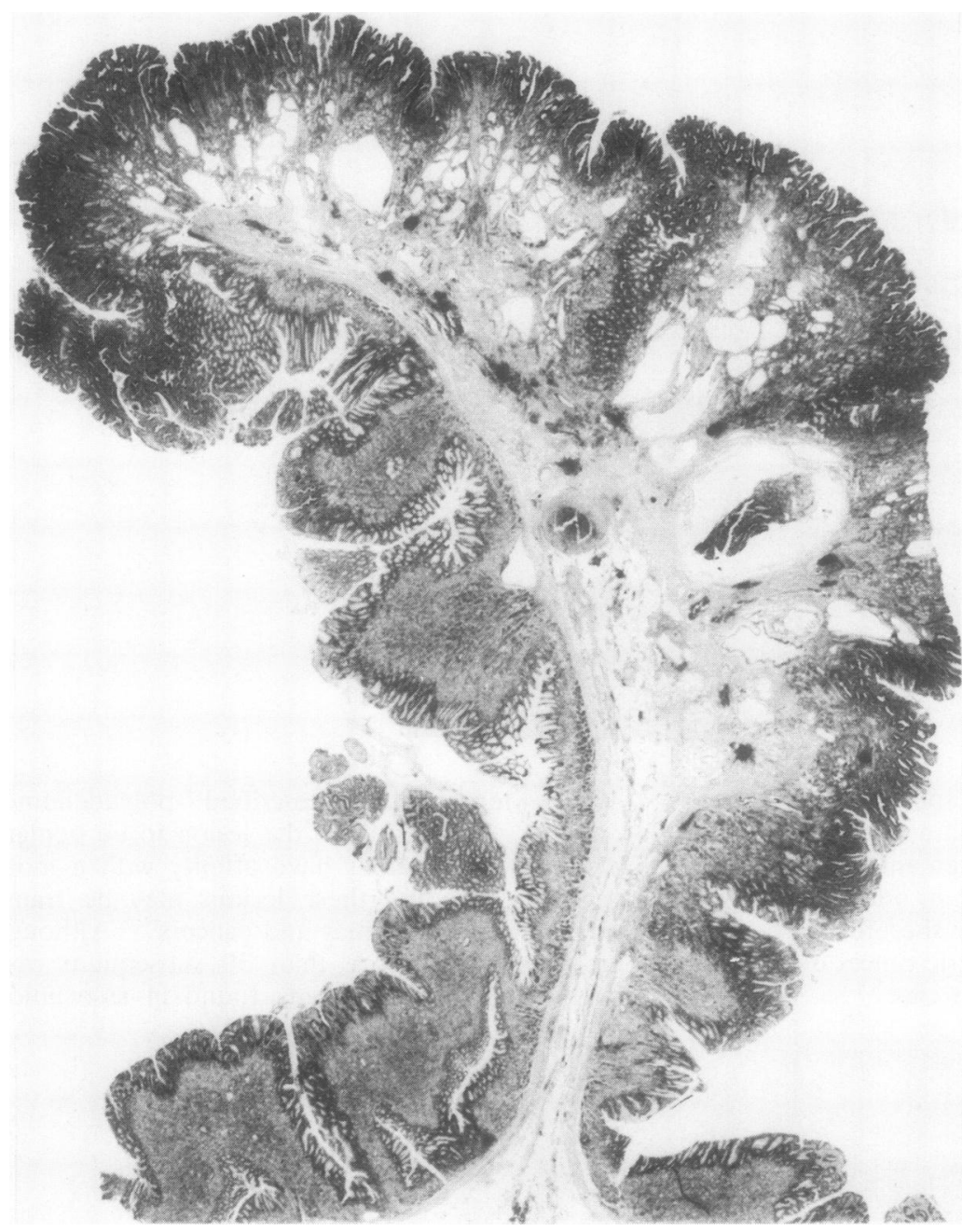

Fig. 4 Giant fold from the gros specimen showing heaped-up gastric mucosa, with cystic dilatation of basal portions of glands. Haematoxylin and eosiñ $\times$ 4.

with giant gastric folds, controversy still exists as to whether there is an increased risk of malignancy in Menetrier's disease. This is mainly because many of the case reports do not document clearly the development of carcinoma during follow-up of histologically proven Menetrier's disease. In some reports the diagnosis of Menetrier's disease was made by radiology or endoscopy, and not confirmed histologically. ${ }^{89}$ In others the histological details are either scanty ${ }^{10}$ or show the presence of conditions other than Menetrier's disease. ${ }^{11}$ In many reports the diagnoses of gastric cancer and Menetrier's disease were either made simultaneously, or made within 12 months of each other, so that the development of carcinoma as a complication of Menetrier's disease cannot be accepted as proven. ${ }^{12-15}$

There appear to be only two previous reports of patients developing carcinoma more than 12 months after the diagnosis of Menetrier's disease was made. ${ }^{1617}$ These patients both died of metastatic gastric cancer five and five and a half years respectively after the diagnosis of Menetrier's disease was made. The present case is thus unique in that follow-up endoscopy and biopsy allowed the detection of gastric cancer at a potentially curable stage.

Due to the rarity of Menetrier's disease, it is unlikely that clearer data on the risk of carcinoma will ever become available. From our experience, we recommend annual endoscopy and biopsy in cases of Menetrier's disease managed medically. Biopsies should be taken from the antrum and body of the 

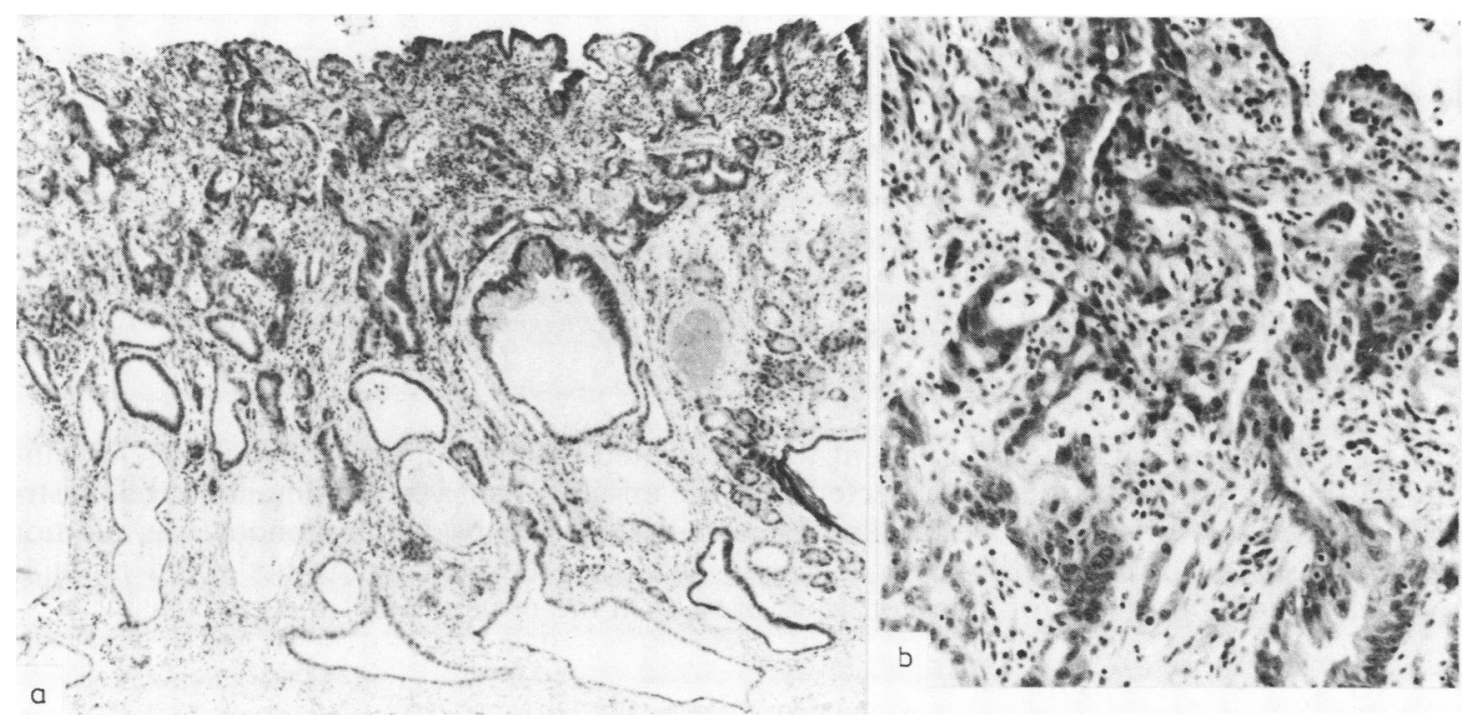

Fig. 5 Low power view (a) and high power view (b) of the antral biopsy from the gastrectomy specimen demonstrating intramucosal carcinoma spreading superficially and features of Menetrier's disease. Haematoxylin and eosin $(a) \times 80(b) . \times$ 200.

stomach, and should be taken both from areas of stomach macroscopically affected by the Menetrier's disease and from apparently normal mucosa. Patients who have had partial gastric resections for Menetrier's disease should be followed-up similarly. No cases have yet been described of gastric carcinoma developing in the gastric stump after surgery for Menetrier's disease, though the published follow'-up data is scanty.

\section{References}

' Scharschmidt BF. The natural history of hypertrophic gastropathy (Menetrier's disease). Am J Med 1977;63:644-52.

${ }^{2}$ Cooper BT, Chadwick VS. Menetrier's Disease. In: Baron JH, Moody FG, eds. Foregut. London: Butterworth \& $\mathrm{Co}$, 1981:141-91.

${ }^{3}$ Butz WC. Giant hypertrophic gastritis. Gastroenterology 1960;39:183-90.

4 Steigmann F, Hyman S, Kannapel WL. Large gastric rugae: benign or malignant. Gdstroenterology 1957;32:72-84.

${ }^{5}$ Moeller DD, Dunn GD, Laing RR, Klotz AP. Hypertrophic gastropathy and carcinoma of the stomach. Gastrointest Endosc 1970;16:159-63.

- Okabe H. Growth of early gastric cancer. In: Murakami T, ed. Early gastric cancer. Gann 1971;11:67-79.

' Menetrier P. Des polyadenomes gastriques et de leurs rapports avec le cancer de l'estomac. Arch Physiol Norm Pathol $1888 ; 1: 32-55 ; 236-62$.

${ }^{8}$ Texter EC, Legerton CW, Reeves RJ, Smith AG, Ruffin JM. Coexistent carcinoma of the stomach and hypertrophic gas- tritis. Report of a case with review of the literature. Gastroenterology 1953;24:579-86.

9 Matzner MJ, Raab AP, Spear PW. Benign giant gastric rugae complicated by submucosal gastric carcinoma. Gastroenterology 1951;18:296-302.

${ }^{10}$ Palumbo LT, Rugtiv GM, Cross KR. Giant hypertrophic gastritis: its surgical and pathological significance. Ann Surg 1951;134:259-67.

"Mills GP. Multiple polyps of the stomach (gastritis polyposa) with report of a case. Br J Surg 1922;10:226-31.

${ }^{12}$ Stamatakis JD. Menetrier's disease and carcinoma of the stomach. Proc R Soc Med 1976;69:264-5.

${ }^{13}$ Vazquez ME, Vazquez IJL, Campo MJ, Valbuena RL. Gastropatia hipertrofica-hiperplasica (enfermedad de Menetrier) asociada a carcinoma gastrico superficial: presentacion de un caso. Rev Esp Enferm Apar Dig (Madrid) 1978;54:173-82.

14 Rubin RG, Fink H. Giant hypertrophy of the gastric mucosa associated with carcinoma of the stomach. Am J Gastroenterol 1967;47:379-88.

is Sanner CJ, Saltzman DA, Mueller JC. Polypoid gastritis. Report of a case associated with gastric adenocarcinoma and review of the literature. Am J Dig Dis 1978;23:19s-24s.

${ }^{16}$ Chusid EL, Hirsch RL, Colcher H. Spectrum of hypertrophic gastropathy. Arch Intern Med 1964;114:621-8.

17 Von Loewenthal M, Steinitz H, Friedlander E. Gastritis hypertrophica gigantea und magenkarzinom. Gastroenterologia (Basel) 1960;93:133-44.

Requests for reprints to: Dr GM Wood, Department of Medicine, St James's University Hospital, Leeds LS9 7TF, England. 